the severity of the coronary lesions was assessed by Gensini scoring system, a method that assigns a different severity score depending on the degree of luminal narrowing and the geographical importance of their locations. 25 patients scored $-40,26$ patients scored 41-80, 23 cases scored $81-120$, and 11 cases scored greater than 120.3 . The results of the retinal vascular measurement and calculation: The mean retinal arteriole diameter (upper temporal branch and inferior temporal branch) in CAD group was significantly smaller than that in control group $(p<0.05)$. The mean retinal venule diameter (upper temporal branch and inferior temporal branch) in CAD group was significantly greater than that in control group $(p<0.05)$. The mean retinal AVR (both in upper temporal branch and inferior temporal branch) in $\mathrm{CAD}$ group was much smaller than that in control group $(p<0.05)$. 4. The correlation analysis between retinal vascular diameter, AVR and the severity of coronary atherosclerosis: the numerical values of retinal artery diameter and AVR in patients with Gensini score group $81-120$ and $>120$ were significantly smaller than that with Gensini score $\leqq 40(p<0.05)$, but the calibre of retinal venule in patients with Gensini score group 81-120 and $>120$ was significantly greater than that with Gensini score $\leqq 40$ $(p<0.05)$, there were no significant differences between the other groups. With Pearson correlation analysis to analyse the correlation of retinal AVR to Gensini score, the result showed that in CAD patients, the retinal AVR was negatively correlated to the Gensini score $(p<0.01)$, With partial correlation analysis and controlling of other influencing factors, such as hypertension and diabetes, the negtive correlation didn't change.

Conclusion In our study, the retinal vascular diameter and AVR are well correlated to the severity of coronary artery disease. In CAD patients, the retinal arteriole calibre and AVR are significantly negatively correlated to Gensini score, and the retinal venule diameter is strongly positively correlated to Gensini score.

\section{e0393 SIGNIFICANCE OF OXIDISED LOW-DENSITY LIPOPROTEININ CORONARY ATHEROSCLEROTIC HEART DISEASE}

doi:10.1136/hrt.2010.208967.393

Xiaoping Meng, Chang Li. Jilin University No 2 Hospital

Objective In this study, control group are people with normal coronary arteries. This study is to discuss the relationship between $\mathrm{LDL}, \mathrm{OX}-\mathrm{LDL}$ and control group, SAP group, UAP group, AMI guoup and contaction of LDL and OX-LDL.

Methods Experimental group are $300 \mathrm{CHD}$ patients without taking lipid-lowering drugs in one month who admittde in the depatrment of cardiolysis from August 2008 to August 2009. They are divided into SAP group (100 cases), UAP group (100 cases), AMI (100 cases). The control group are 100 cases of patients who are randomly selected and confirmed without CHD by coronary angiography in the same period in our department. Information includs gender, age, smoking, drinking, hypertension, diabetes, levels of LDL and OX-LDL. We analysis patients' basic information, the level and correlation of LDL and OX-LDL in control group, SAP group, UAP group and AMI group.

Results 1 . There are no significant differents between control group and CHD groups in basic information ( $p>0.05)$. 2. Concentration of $\mathrm{LDL}$ in CHD group is significant difference compared with control group $(p<0.01)$. Concentration of LDL in UAP group is no significant difference compared with SAP group ( $p>0.05)$. Concentration of LDL in AMI group is significant difference compared with SAP group $(p<0.01)$. Concentration of LDL in AMI group is no significant difference compared with UAP group $(p>0.05)$. 3. Concentration of OX-LDL in CHD group is significant difference compared with control group $(p<0.01)$. Concentration of OX-LDL in UAP group is significant difference compared with SAP group $(p<0.05)$.
Concentration of OX-LDL in AMI group is significant difference compared with SAP group $(p<0.01)$. Concentration of OX-LDL in AMI group is significant difference compared with UAP group $(p<0.01)$. 4. There is no correlation between concentration of LDL and OX-LDL in all groups ( $\mathrm{p}>0.05)$.

Conclusions 1 . We confirm that LDL and OX-LDL are risk factors for $\mathrm{CHD}$. There is no significant differences about the concentration of $\mathrm{LDL}$ in CHD groups, however, there is significant differences about the concentration of OX-LDL in CHD groups. The level of OX-LDL is in escalating trend. 2 There is no correlation between concentration of LDL and OX-LDL in all groups $(p>0.05)$ and OX-LDL play a more important role in the process of CHD. Compared with LDL, mensurating OX-LDL is more meaningful in the treatment and prevention of $\mathrm{CHD}$.

\section{e0394 THE RELATIONSHIP BETWEEN OBSTRUCTIVE SLEEP APNEA AND ENDOTHELIN-1 PLASMA LEVELS IN PATIENTS WITH CORONARY HEART DISEASE}

doi:10.1136/hrt.2010.208967.394

Zhao Qing, Liu Zhihong, Zhao Zhihui, Luo Qin, Zhang Hongliang, Wang Yong. Fuwai Hospital, Beijing, China

Objective Obstructive sleep apnea (OSA) is an independent risk factor for cardiovascular morbidity and mortality. The mechanism is unknown, but recent studies provide evidence that endothelial dysfunction might contribute. So we investigate the relationship between obstructive sleep apnea and endothelin-1 (ET-1) plasma levels in patients with coronary heart disease.

Methods 287 patients with coronary heart disease were enrolled and an overnight polysomnography was performed to all of them. According to the apnea-hypopnoea index, the patients were divided into four groups: no OSA group ( $\mathrm{AHI}<5, \mathrm{n}=58$ ), mild OSA group $(5 \leq \mathrm{AHI}<15, \mathrm{n}=82)$, moderate OSA group $(15 \leq \mathrm{AHI}<30, \mathrm{n}=70)$, severe OSA group (AHI $\geq 30, n=77$ ). Plasma levels of endothelin-1 were detected to all the patients.

Results Compared with no and mild OSA group, endothelin-1 plasma levels in severe OSA group elevated significantly $(p=0.009)$, even after analysis in a general linear model with correction for confounders. Plasma ET-1 levels showed an increasing trend within no OSA, mild OSA and moderate OSA group, however no statistically significant was observed between no OSA group and patients with mild or moderate OSA, respectively ( $p=0.421, p=0.226$ ).

Conclusion Among patients with coronary heart disease, plasma ET-1 levels elevated significantly in severe OSA group. This might support that severe OSA has more effects on endothelium function for coronary heart disease than mild and moderate OSA.

\section{e0395 CORRELATION BETWEEN SERUM LEVELS OF CATHEPSIN S AND SEVERITY OF ATHEROSCLEROSTIC LESIONS OF CORONARY ARTERIES IN CHINESE}

doi:10.1136/hrt.2010.208967.395

Liu Yu-sheng, Hao Lin, Jiang Wei-dong, Lu Qing-hua, Wang Yong- Mei. Department of Cardiology, The Second Hospital of Shandong University, Jinan

Objective Previous studies have showed that cathepsin S(CatS) is upregulated in atherosclerosis lesions in humans. We try to deplore the association between serum levels of CatS and its inhibitor cystatin $\mathrm{C}(\mathrm{Cys} \mathrm{C})$ and the severity of atherosclerostic lesions of coronary arteries in Chinese.

Methods and results 107 coronary atherosclerositic diseases (CAD) and 48 controls were recruited and all subjects consisting were verified by selective angiography, the the severity of lesions of 